\title{
Outcomes of primary fusion in high-energy Lisfranc injuries at a tertiary state hospital
}

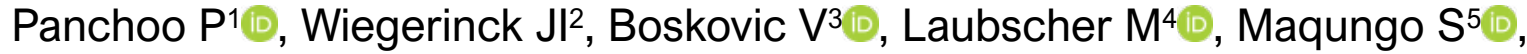 \\ McCollum G6 ${ }^{6}$, Dey $R^{7}$ (i)
}

1 MBChB; Orthopaedic Registrar, Department of Orthopaedics Surgery, Groote Schuur Hospital, University of Cape Town, South Africa

$2 \mathrm{MD}$, PhD; Consultant, Department of Orthopaedics, Joint Research, OLVG Hospital Amsterdam, The Netherlands

3 MBChB; Orthopaedic Registrar, Department of Orthopaedics, Groote Schuur Hospital, University of Cape Town, South Africa

${ }^{4}$ MBChB(UFS), Dip PEC, FC Orth(SA), MMed(Orth)(UCT); Consultant, Department of Orthopaedics, Groote Schuur Hospital, University of Cape Town, South Africa

5 MBChB(Natal), FC Orth(SA), MMed(Orth)(UCT); Consultant, Department of Orthopaedics, Groote Schuur Hospital, University of Cape Town, South Africa

6 MBChB, MMed(UCT), FC Orth(SA); Consultant, Department of Orthopaedics, Groote Schuur Hospital, University of Cape Town, South Africa

7 PhD; Post-doctoral Fellow, Faculty of Health Sciences, University of Cape Town, South Africa; Groote Schuur Hospital, University of Cape Town, South Africa

Corresponding author: Dr P Panchoo, Department of Orthopaedics, Old Main Building, Groote Schuur Hospital, Anzio Road, Observatory, Cape Town, 7925, South Africa; tel: +27 (82) 815 0238; email: praveshpanchoo@gmail.com

\section{Abstract}

Background: High-energy Lisfranc injuries are relatively uncommon but can lead to severe disability and morbidity. Primary fusion is a treatment option that can improve outcomes and reduce the reoperation rate. The aim of this study was to evaluate our series of primary fusions for high-energy Lisfranc injuries, looking specifically at type of fusion, time to union, non-union rates, reoperation rates and quality of reduction.

Methods: Patients who underwent surgery for Lisfranc injuries were identified from the REDCap surgical database and then retrieved from records. Only cases of primary fusion in adults were included. We excluded low-energy sprains and athletic injuries, ipsilateral lower limb injuries and cases where reduction and fixation were done. Radiographs were analysed from the iSite Enterprise PACS system (Philips ${ }^{\circledR}$ ).

Results: Between 2013 and 2018, 12 cases of high-energy Lisfranc injuries were identified where primary fusion was done. Seven patients (58\%) underwent fusion of the first, second and third tarsometatarsal (TMT) joints. The first and second TMT joints were fused in only one case (8\%), and the second and third TMT joints were fused in four cases (33\%). Only one patient (8\%) had removal of implants. Compression plating was the technique of choice used for fusion. There was $100 \%$ union rate and average time to union was 84 days. Acceptable reduction was observed in nine cases (75\%). Three cases (25\%) of malreduction were found, among which one patient had pre-existing hallux valgus.

Conclusion: The majority of patients who underwent primary fusion of at least one TMT joint had good radiological outcome. Further studies with better clinical follow-up are needed.

Level of evidence: Level 4

Keywords: Lisfranc, tarsometatarsal, outcome, fusion

Citation: Panchoo P, Wiegerinck JI, Boskovic V, Laubscher M, Maqungo S, McCollum G, Dey R. Outcomes of primary fusion in high-energy Lisfranc injuries at a tertiary state hospital. SA Orthop J 2020;19(3):150-155. http://dx.doi.org/10.17159/2309-8309/2020/v19n3a4

Editor: Prof. NP Saragas, University of the Witwatersrand, Johannesburg, South Africa

Received: October 2019

Accepted: March 2020

Published: August 2020

Copyright: ( 2020 Panchoo P. This is an open-access article distributed under the terms of the Creative Commons Attribution Licence, which permits unrestricted use, distribution and reproduction in any medium, provided the original author and source are credited.

Funding: No external funding was received for this study.

Conflict of interest: The authors declare they have no conflicts of interest that are directly or indirectly related to the research. 


\section{Introduction}

Lisfranc fracture dislocations also known as tarsometatarsal (TMT) fracture dislocation, consist of injuries to the bases of the five metatarsals, their articulations with the four distal tarsal bones, and disruption of the Lisfranc ligamentous complex. ${ }^{1-4}$ The main stabilising ligament of the midfoot (the Lisfranc ligament) runs on the plantar aspect of the foot from the lateral aspect of the medial cuneiform to the medial aspect of the base of the second metatarsal. ${ }^{1,3}$ It functions to stabilise the TMT articulation of the foot. Injury to the ligament and the TMT complex can lead to chronic pain, midfoot arthritis, decreased function, and loss of quality of life..$^{1,5-7}$ These injuries include any combination of bony and ligament disruption to this complex, hence classifying the pattern of injury can be difficult. Computer tomography (CT) scans are therefore invaluable and virtually mandatory for all Lisfranc injuries. This is a relatively uncommon injury with an incidence of approximately $0.2 \%$ of all fractures. ${ }^{1,6,8}$ Often Lisfranc injuries are missed in a polytraumatised patient, or in low-energy athletic injuries leading to poor functional outcomes.

Historically they have been associated with high-energy mechanisms such as motor vehicle accidents, falls or crush injuries. ${ }^{4,6,8}$ The treatment of these high-energy injuries has evolved over time with authors now agreeing that an anatomic reduction through open surgery is of critical importance. ${ }^{1-8}$ Recently, more research on Lisfranc injuries has focused on relatively low-energy mechanisms and Lisfranc injuries in athletes. ${ }^{7}$

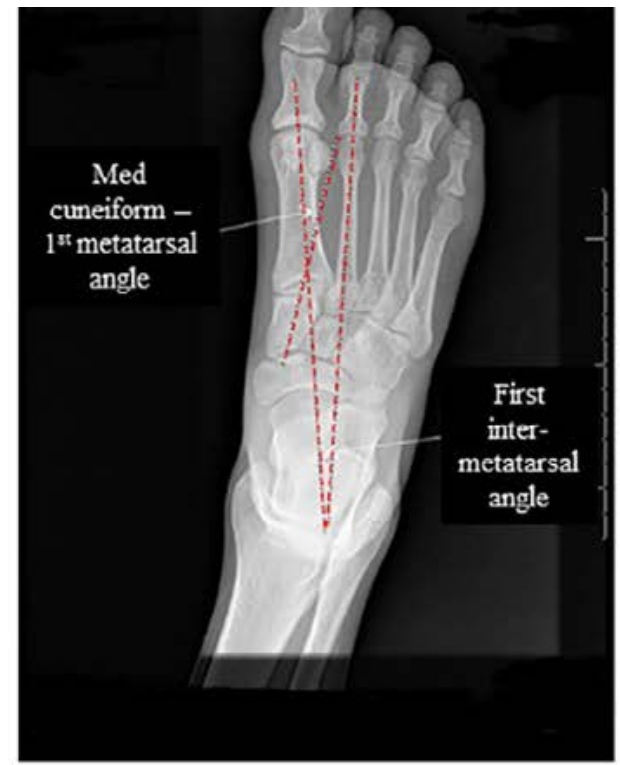

(a)
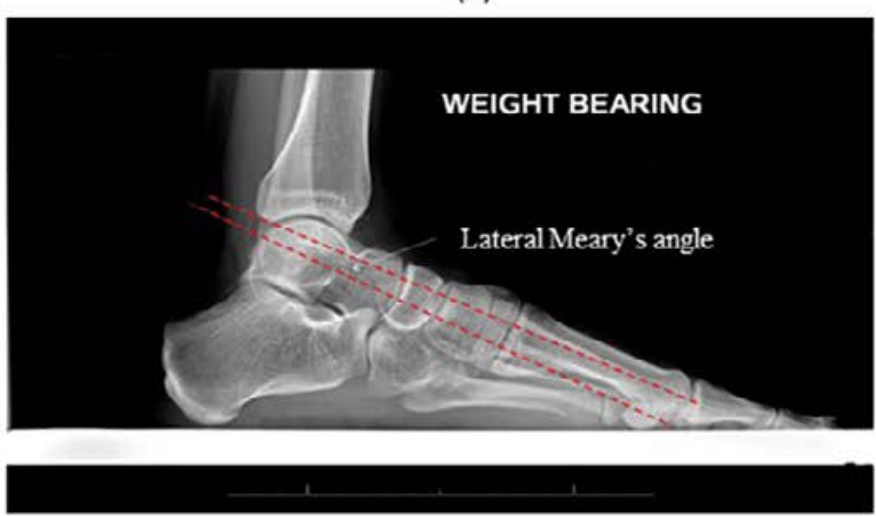

(c)
Anatomic reduction and rigid stabilisation are known to be the standard of care. ${ }^{1-8}$ Both open reduction and internal fixation (ORIF) and primary fusion have been evaluated as treatment options. With ORIF there is a high reoperation rate to remove the internal fixation or to perform a fusion of the midfoot due to ongoing midfoot arthritis. ${ }^{1-6}$ This has led some authors to believe that a primary fusion should be performed in the first setting. In a systematic review in 2012, Sheibani-Rad et al. ${ }^{9}$ concluded that both methods were acceptable, but no consensus was reached as to which is superior.

The aim of this study is to evaluate our series of primary fusions for high-energy Lisfranc injuries, looking specifically at time to union, non-union rates, reoperation rates and quality of reduction.

\section{Methods}

After local institution review board approval, patients who underwent primary fusion for Lisfranc injuries at Groote Schuur Hospital (GSH) between January 2013 and December 2018 were identified. Post-operative radiographs at six weeks, three months and six months were reviewed by two independent reviewers, JIW and PP, assessing quality of reduction, union rate and removal of implants. All radiographs were taken weight bearing and according to international standards. JIW is an orthopaedic surgeon from the Netherlands with two years of consultant experience. PP is a thirdyear orthopaedic registrar at GSH. All measurements were further verified by the supervisor GM who is head of the foot and ankle department.

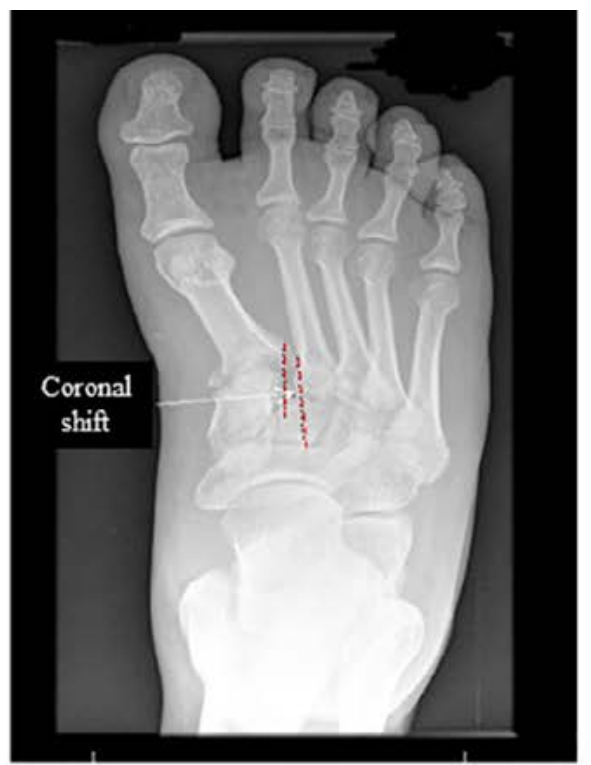

(b)

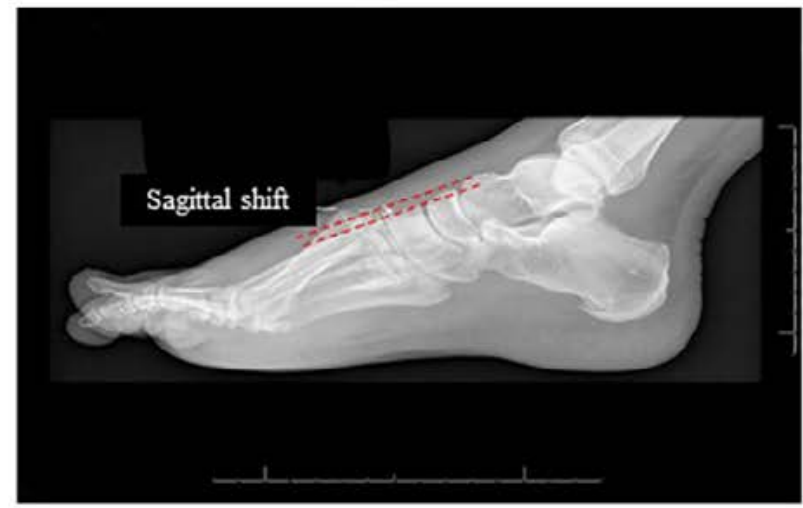

(d)

Figure 1. (a) First intermetatarsal angle (IMA) and medial cuneiform-first metatarsal angle; (b) coronal shift; (c) lateral Meary's angle; and (d) sagittal shift 
Table I: Definition and normal range of measured parameters

\begin{tabular}{|l|l|l|}
\hline $\begin{array}{l}\text { Anteroposterior (AP) and lateral weight-bearing } \\
\text { radiographs }\end{array}$ & Definition ${ }^{11,12}$ \\
\hline First intermetatarsal angle (AP view) & $\begin{array}{l}\text { Angle formed by the line drawn bisecting the first metatarsal and } \\
\text { bisecting the second metatarsal }\end{array}$ \\
\hline Medial cuneiform-first metatarsal angle (AP view) & $\begin{array}{l}\text { Angle formed by the bisection of the medial cuneiform and the } \\
\text { anatomic axis of the first metatarsal }\end{array}$ \\
\hline Lateral Meary's angle (lateral view) & $\begin{array}{l}\text { Angle formed by the bisection of the talar neck and the anatomic } \\
\text { axis of the first metatarsal }\end{array}$ \\
\hline Sagittal shift (lateral view) & $\begin{array}{l}\text { Superior translation of second metatarsal with respect to the } \\
\text { anterior cortex of the middle cuneiform }\end{array}$ \\
\hline Coronal shift (AP view) & $\begin{array}{l}\text { Lateral translation of the second metatarsal with respect to the } \\
\text { medial cortex of the middle cuneiform }\end{array}$ \\
\hline
\end{tabular}

Cases of high-energy Lisfranc injuries were retrieved from the orthopaedic surgery database REDCap ${ }^{\circledR}$, and corresponding radiographs were analysed from our local picture archiving and communication system, iSite Intellispace ${ }^{\circledR}$ software.

Injuries were classified using the Myerson classification of Lisfranc injuries. ${ }^{10}$ Union was assessed by the formation of crosstrabeculation and the absence of fracture line on two views. Quality of reduction was assessed by measuring the following parameters ${ }^{11,12}$ on AP view: first intermetatarsal angle (IMA), medial cuneiform-first metatarsal angle, coronal shift. On lateral view: talus-first metatarsal angle (lateral Meary's angle), sagittal shift (Figure 1). Any value outside of the normal range ${ }^{11,12}$ (Table I) was qualified as a malreduction/malunion. Cases of implant removal and reoperation were obtained from patients' files.

\section{Surgical technique}

A CT scan was performed prior to surgery in all patients to assess fracture displacement and intra-articular comminution. The surgical technique used is a dorsal two-incision approach to gain access to the medial and middle columns. The medial column incision is placed dorsally over the first TMT joint just medial to the extensor hallucis longus tendon. The lateral incision is centred between the second and third metatarsal to gain access to the second and third TMT joints and maintain an adequate soft tissue bridge. A third incision between the fourth and fifth metatarsal is used in case open reduction of the fourth and fifth TMT is needed. A single incision over the first TMT joint or between the second and third

a

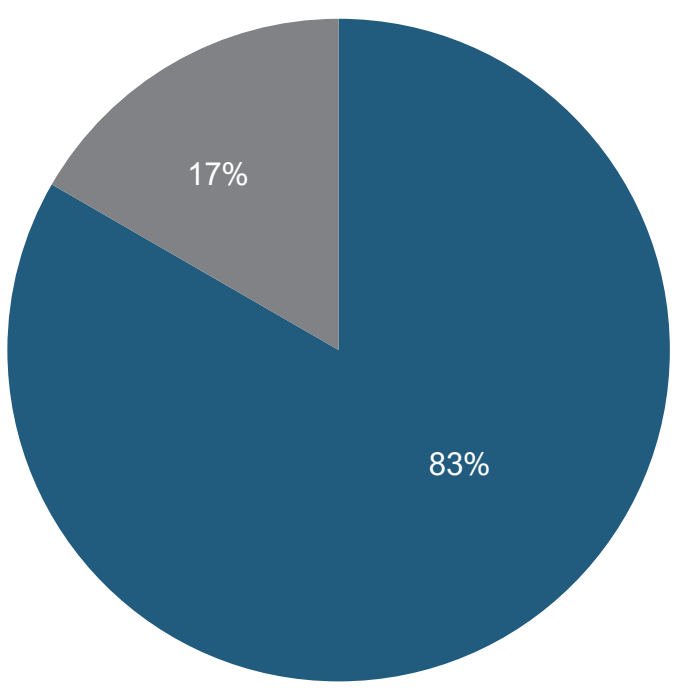

Male

Female
TMT joints is used in cases where the first and second, or second and third, TMT joints are fused. Our method of primary fusion was careful preparation of the joint by debriding the cartilage layer, reduction of the fracture dislocation, bone grafting of the defect with autograft taken from ipsilateral calcaneus followed by dorsal compression plating. The number of TMT joints fused depended on its involvement in the fracture pattern and its congruency. The first, second and third TMT joints were considered for fusion in the case of intra-articular comminution and joint incongruency. Fourth and fifth TMT joints were never considered for fusion; no cases of intra-articular fractures involving these joints were identified. Subluxation of the fourth and fifth TMT joints were addressed with temporary Kirschner wires removed at six weeks. All surgeries were performed by the foot-and-ankle consultant as primary surgeon. Patients were immobilised in a below-knee circular cast for six weeks and kept non-weight bearing for at least 12 weeks until radiological evidence of union.

\section{Results}

Between 2013 and 2018, 17 cases of Lisfranc injuries were identified. Twelve of them underwent primary fusion and five cases were excluded (in one case the file could not be retrieved, two were cases of closed reduction and percutaneous screw fixation, and two underwent open reduction and internal fixation). The study group comprised ten male and two female patients (Figure 2a), with a mean age of 38.9 years (range 18-60) (Figure 2b). All patients were involved in high-energy injury mechanisms, the commonest being

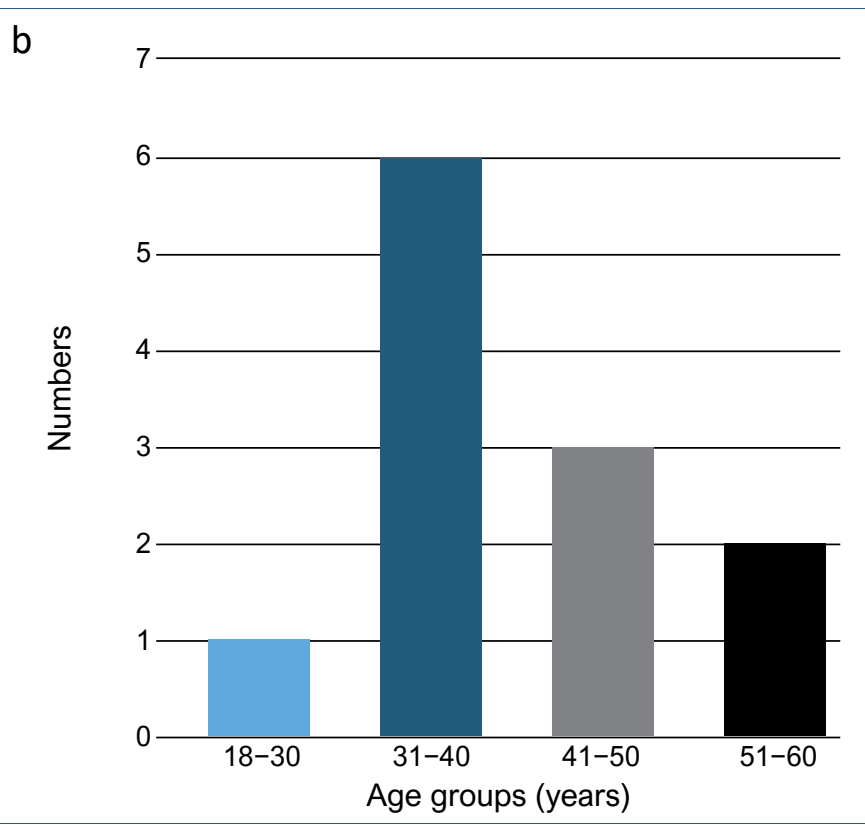

Figure 2. (a) Sex demographics of the patient group used for this study; (b) distribution of patients in various age groups 


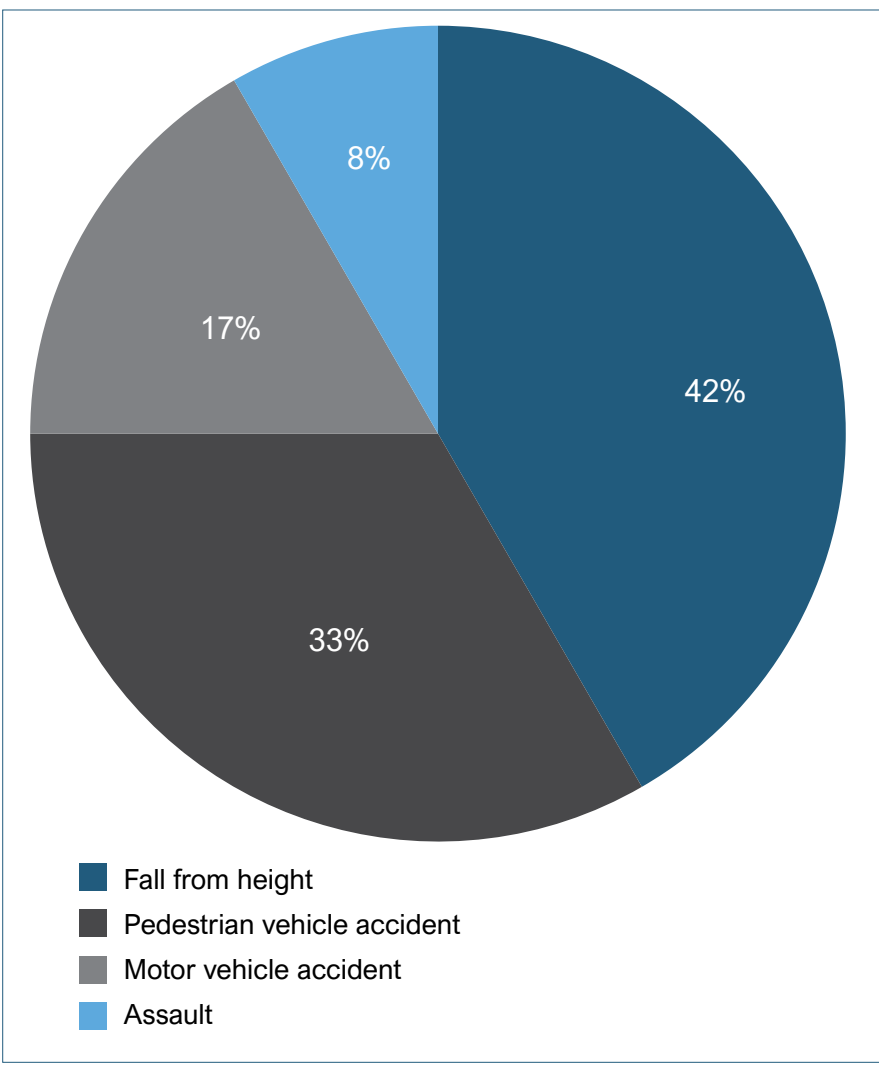

Figure 3. Mechanism of injury

fall from height, followed by pedestrian vehicle accident (Figure 3).

Most of our cases were Myerson ${ }^{10}$ type A lateral, representing $66 \%$. The remaining were one case each of type A medial, B1, B2 and $\mathrm{C} 2$ respectively.

Seven patients (58\%) underwent fusion of the first, second and third TMT joints; the first and second TMT joints were fused in only one case $(8 \%)$ and the second and third TMT joints fused in four cases (33\%). Acceptable reduction was observed in nine cases (75\%); three cases $(25 \%)$ of malreduction were noted among which one patient had pre-existing hallux valgus which can give false negative values in measuring the IMA. Our measured reduction parameters for all the patients is shown in Table II. Average reduction parameters in the well-reduced group were as follows (Table III): IMA of $8.05^{\circ}$, medial cuneiform-first metatarsal angle of $16.9^{\circ}$, lateral Meary's angle $5.9^{\circ}$, coronal shift $0.7 \mathrm{~mm}$. Only one patient had a sagittal shift of $1.6 \mathrm{~mm}$; the remaining radiographs had no sagittal shift. Average time to union in our series was 84 days, and no case of non-union was identified (100\% union rate). We also compared the number of TMT joints fused to the quality of reduction (Table IV). We found better results in cases where either the first, second and third metatarsals (86\%) or second and third metatarsals were fused (75\%). There was one case of fusion of only first and second metatarsals which was malreduced. One patient (8\%) had removal of implants for prominent hardware.

\section{Discussion}

The most common complication following ORIF is post-traumatic osteoarthritis (PTOA) with rates estimated between $20 \%$ and $50 \% .{ }^{13}$ Teng et al. ${ }^{14}$ performed gait analysis on patients following ORIF and demonstrated, despite restoration of normal anatomy and gait mechanics, patients still had poor American Orthopedic Foot and Ankle Score (AOFAS) midfoot scores. In the setting of PTOA and continued pain, secondary arthrodesis was used as a salvage procedure. In a prospective randomised study, Ly and Coetzee $^{15}$ compared ORIF versus primary fusion in primarily ligamentous Lisfranc injuries. Seventy-five per cent of patients who underwent ORIF had some loss of correction and degenerative joint changes at the final follow-up visit at a mean of 42.5 months post-operatively. They concluded that primary fusion provides

Table III: Average reduction parameters

\begin{tabular}{|l|c|c|}
\hline & $\begin{array}{c}\text { Normal } \\
\text { range }\end{array}$ & Average measured value \\
\hline $\begin{array}{l}\text { First intermetatarsal } \\
\text { angle }\end{array}$ & $6-10^{\circ}$ & $8.1^{\circ}$ \\
\hline $\begin{array}{l}\text { Medial cuneiform- } \\
\text { first metatarsal angle }\end{array}$ & $16-20^{\circ}$ & $16.9^{\circ}$ \\
\hline Lateral Meary's angle & $2-10^{\circ}$ & $6.0^{\circ}$ \\
\hline Sagittal shift & $<2 \mathrm{~mm}$ & Only one case of $1.6 \mathrm{~mm}$ shift \\
\hline Coronal shift & $<2 \mathrm{~mm}$ & 0.7 \\
\hline
\end{tabular}

Table IV: Effect of the number of bones fused on the quality of reduction

\begin{tabular}{|l|c|c|c|}
\hline Fused bones & $\begin{array}{c}\text { Number } \\
\text { of cases }\end{array}$ & $\begin{array}{c}\text { Improper } \\
\text { reduction }\end{array}$ & $\begin{array}{c}\text { Proper } \\
\text { reduction }\end{array}$ \\
\hline First, second and third TMT & 7 & $1(14 \%)$ & $6(86 \%)$ \\
\hline First and second TMT & 1 & $1(100 \%)$ & 0 \\
\hline Second and third TMT & 4 & $1(25 \%)$ & $3(75 \%)$ \\
\hline
\end{tabular}

Table II: Measurements for all patients (the well-reduced cases are highlighted in blue and the malreduced cases in yellow)

\begin{tabular}{|c|c|c|c|c|c|}
\hline Patient no. & IMA (degrees) & $\begin{array}{c}\text { Medial cuneiform-first metatarsal } \\
\text { angle (degrees) }\end{array}$ & Meary's angle (degrees) & Sagittal shift (mm) & Coronal shift (mm) \\
\hline 1 & 5.7 & 16.5 & 5.6 & 0 & 0 \\
\hline 2 & 6.2 & 16.1 & 6.9 & 0 & 0 \\
\hline 3 & 9.3 & 17.4 & 5.5 & 0 & 1.2 \\
\hline 4 & 8.5 & 16.1 & 8.2 & 0 & 0.8 \\
\hline 5 & 8.9 & 16.2 & 8.6 & 1.6 & 1.4 \\
\hline 6 & 6.9 & 17.6 & 8.6 & 0 & 0 \\
\hline 7 & 9.4 & 19.8 & 4.3 & 0 & 1.5 \\
\hline 8 & 9.8 & 16.2 & 2.1 & 0 & 0 \\
\hline 9 & 7.8 & 16.5 & 3.8 & 0 & 1.5 \\
\hline 10 & 20.9 & 26.9 & 5.8 & 0 & 1.5 \\
\hline 11 & 12.4 & 30.2 & 7.5 & 1.0 & 1.2 \\
\hline 12 & 13.5 & 23.0 & 13.6 & 4.2 & 0 \\
\hline
\end{tabular}




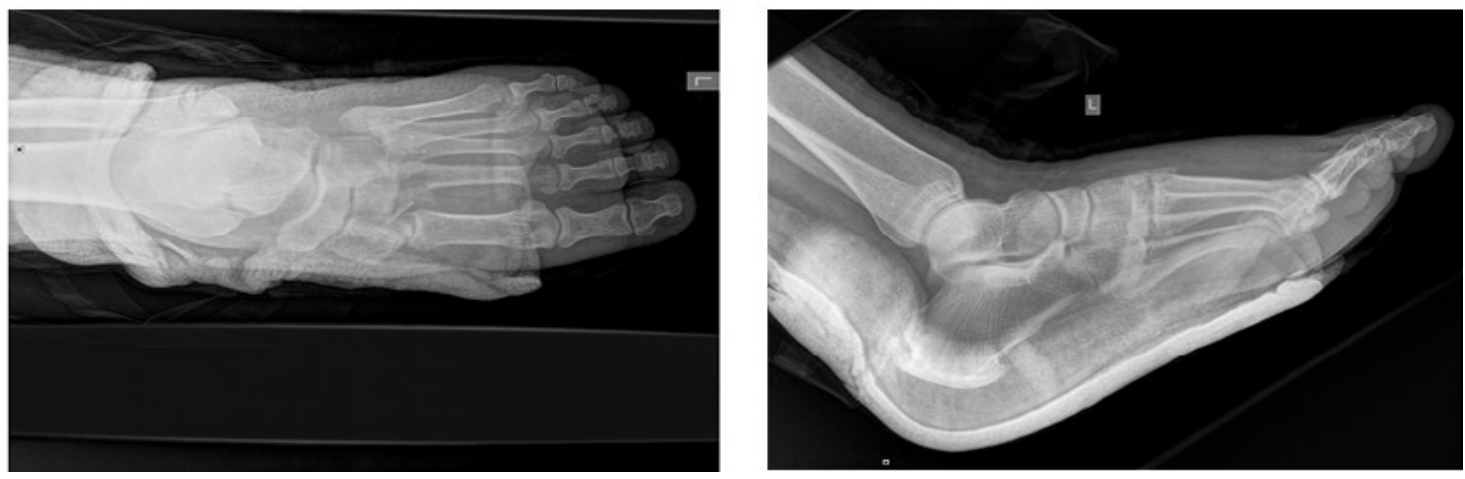

Pre-operative
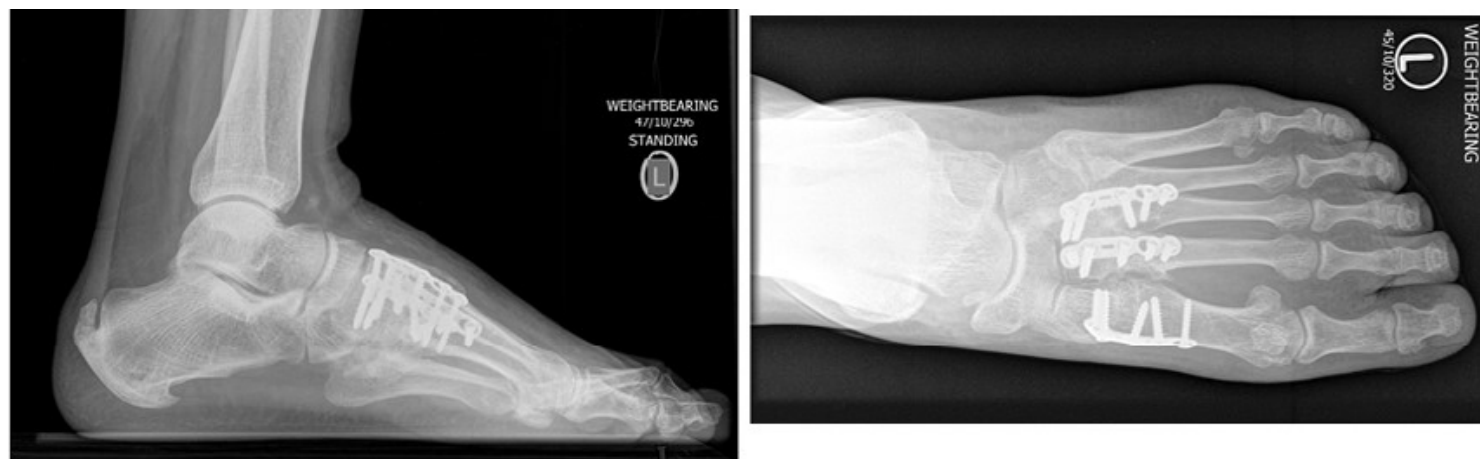

Six months post-operative

Figure 4. Pre- and post-reduction radiographic evidence of a well-reduced Lisfranc injury

better short- and medium-term outcomes than ORIF for primarily ligamentous injuries. Sheibani-Rad et al. ${ }^{9}$ performed a qualitative, systematic review comparing primary fusion and ORIF and found six reports with a combined total of 193 patients. The one-year follow-up AOFAS score for the ORIF group was 72.5 versus 88.0 for the primary fusion group. They concluded that both procedures yield satisfactory results, but primary fusion might have a slight advantage in clinical outcomes. ${ }^{9,16}$ In most of these studies, the complexity and energy of the injury has not been stated. Lowenergy athletic injuries have been grouped with high-energy injuries, which we believe have a different prognosis if a primary fusion is not performed.

Our study did not compare outcome of ORIF versus primary fusion, but we analysed the results of patients who had primary fusion in high-energy Lisfranc fractures. As described in the literature, our series of patients showed good results in terms of reduction (75\%) and reasonable time to union ( 84 days). Twentyfive per cent of malreduction was observed but this figure did not necessarily correlate with a poor clinical result. Despite much effort to trace the patients, only two responded and came for follow-up. We calculated the AOFAS scores for these two patients and both scored $90 / 100$. The remaining patients were untraceable. Only one case of implant removal (8\%) was identified due to symptomatic hardware. The latter correlated with the low revision or repeat surgery described in literature. Better quality of reduction was observed with fusion of the first, second and third TMT and second and third TMT joints ( $86 \%$ and $75 \%$ respectively). The first TMT joint frequently involves primarily ligamentous injury with minimal fractures of the first metatarsal base and medial cuneiform. Fusion of the first TMT joint stabilises the medial column and acts as a buttress to further stabilise adjacent fracture-dislocation injuries. ${ }^{16}$ A reason for the $25 \%$ malreduction rate can be attributed to significant comminution, bone loss and gross instability. There may have been some cases where the medial column was unstable but not included in the fusion leading to worsening of the reduction parameters mentioned. Figure 4 illustrates a case of high-energy Lisfranc injury operated at GSH with fusion of the first, second and third TMT joints appropriately reduced.

In conclusion, our data suggests that primary fusion may be a viable option in these high-energy Lisfranc injuries with good radiological outcome, $100 \%$ union and low reoperation rate. The malreduction rate may be improved by including the medial column in the fusion construct. Our study was limited by a small population size and the lack of clinical parameters to compare with the radiological results. However, this is a short-term study and the long-term consequences of arthrodesis in young, athletic patients remain unknown. Long-term, prospective studies of highenergy injuries with adequate clinical follow-up would be beneficial to establish and evaluate indications for primary fusion in this population.

\section{Ethics statement}

This submission is in accordance with the principles laid down by the Responsible Research Publication Position Statements as developed at the 2nd World Conference on Research Integrity in Singapore, 2010. Prior to commencement of the study, ethical approval was obtained from the following ethical review board: Human Research Ethics Committee, HREC REF: 446/2019. This article does not contain any studies with human participants or animals performed by any of the authors.

\section{Declaration}

The authors declare authorship of this article and that they have followed sound scientific research practice. This research is original and does not transgress plagiarism policies.

\section{Author contributions}

PP: Study conceptualisation, manuscript preparation, data capturing, data analysis, manuscript revision

JIW: Data capture, manuscript revision

VB: Study conceptualisation, manuscript revision

ML: Data capture, manuscript preparation

SM: Data capture, manuscript revision

GM: Study conceptualisation, data capture, manuscript revision

$\mathrm{RD}$ : Data analysis, manuscript revisions

\section{ORCID}

Panchoo P (D) https://orcid.org/0000-0003-1688-9214

Boskovic V iD https://orcid.org/0000-0001-9275-2151 
Laubscher M (D) https://orcid.org./0000-0002-5989-8383 Maqungo S (D) https://orcid.org./0000-0002-8735-8341 McCollum G (D) https://orcid.org./0000-0002-1982-4654 Dey R (D) https://orcid.org/0000-0002-3616-1995

\section{References}

1. Kirzner N, Zotov P, Goldbloom D, Curry H, Bedi H. Dorsal bridge plating or transarticular screws for Lisfranc fracture dislocations a retrospective study comparing functional and radiological outcomes. Bone Joint J. 2018;100-B:468-74. https://doi. org/10.1302/0301-620X.100B4.BJJ-2017-0899.R2.

2. Ponkilainen VT, Laine HJ, Mäenpää HM, Mattila VM, Haapasalo $\mathrm{HH}$. Incidence and characteristics of midfoot injuries. Foot \& Ankle International. 2019;40(1):105-12. https://doi. org/10.1177/1071100718799741.

3. Knijnenberg LM, Dingemans SA, Terra MP, et al. Radiographic anatomy of the pediatric Lisfranc joint. J Pediatr Orthop. 2018;38(10):510-13. https://doi.org/10.1097/BPO.000000000000 0864.

4. Cochran G, Renninger C, Tompane T, Bellamy J, Kuhn K. Primary arthrodesis versus open reduction and internal fixation for low-energy Lisfranc injuries in a young athletic population. Foot \& Ankle International. 2017;38(9):957-63. https://doi. org/10.1177/1071100717711483.

5. Diacon AL, Kimmel LA, Hau RC, Gabbe BJ, Edwards ER Outcomes of midfoot and hindfoot fractures in multitrauma patients. Injury. 2019;50(2):558-63. https://doi.org/10.1016/j. injury.2018.11.021.

6. Pigott MT, Shah R, Chan J, et al. Initial displacement does not affect loss of reduction after Lisfranc fracture dislocations. Foot \& Ankle Specialist. 2019;12(6):535-39. https://doi. org/10.1177/1938640018823067.

7. Hong CC, Pearce CJ, Ballal MS, Calder JD. Management of sports injuries of the foot and ankle. Bone Joint J. 2016;98-B(10):1299 311. https://doi.org/10.1302/0301-620X.98B10.37896.

8. Hawkinson MP, Tennent DJ, Belisle J, Osborn P. Outcomes of Lisfranc injuries in an active duty military population. Foot \& Ankle International. 2017;38(10):1115-119. https://doi.org/10.1177/ 1071100717719532

9. Sheibani-Rad S, Coetzee JC, Giveans MR, DiGiovann C. Arthrodesis versus ORIF for Lisfranc fractures. Orthopedics. 2012;35(6):868-73. https://doi.org/10.3928/014 77447-20120525-26.

10. Stavlas P, Roberts CS, Xypnitos FN, Giannoudis PV. The role of reduction and internal fixation of Lisfranc fracture-dislocations: a systematic review of the literature. Int Orthop. 2010;34(8):1083-91. https://doi.org/10.1007/s00264-010-1101-x.

11. Hatch DJ, Smith A, Fowler T. Radiographic relevance of the dista medial cuneiform angle in hallux valgus assessment. J Foot Ankle Surg. 2015;55(1):85-89. https://doi.org/10.1053/j.jfas.2015.06.026.

12. Gibboney MD, LaPorta GA, Dreyer MA. Interobserver analysis of standard foot and ankle radiographic angles. J Foot Ankle Surg. 2019;58(6):1085-90. https://doi.org/10.1053/j.jfas.2018.12.037.

13. Schepers T, Oprel PP, Van Lieshout EM. Influence of approach and implant on reduction accuracy and stability in Lisfranc fracture-dislocation at the tarsometatarsal joint. Foot \& Ankle Int. 2013;34(5):705-10. https://doi.org/10.1177/1071100712468581.

14. Teng AL, Pinzur MS, Lomasney L, Mahoney L, Havey R. Functiona outcome following anatomic restoration of tarsal-metatarsal fracture dislocation. Foot \& Ankle Int. 2002;23(10):922-26. https:// doi.org/10.1177/107110070202301006.

15. Ly TV, Coetzee JC. Treatment of primary ligamentous Lisfranc joint injuries: primary arthrodesis compared with open reduction internal fixation. J Bone Joint Surg Am. 2006;88(3):514-20. https:// doi.org/10.2106/JBJS.E.00228.

16. Boffeli TJ, Collier RC, Schnell KR Combined medial column arthrodesis with open reduction internal fixation of centra column for treatment of Lisfranc fracture-dislocation: a review of consecutive cases. J Foot Ankle Surg. 2018;57(6):1059-66. https:// doi.org/10.1053/j.jfas.2018.03.026. 